\title{
lleo-ileal intussusception due to small bowel melanoma
}

\author{
Mahteme Bekele Muleta ${ }^{*}, 1$, Desalegn Abdisa ${ }^{1}$, Bereket Berhane ${ }^{2}$ \\ ${ }^{1}$ Department of Surgery, St. Paul's Hospital Millennium Medical College, Addis Ababa, Ethiopia, \\ ${ }^{2}$ Department of Pathology, St. Paul's Hospital Millennium Medical College, Addis Ababa, Ethiopia
}

\begin{abstract}
Gastrointestinal tract malignant melanoma is an uncommon form of neoplasm that may be either primary or metastatic. Diagnosis is often delayed until the illness becomes an emergency due to complications, such as intestinal hemorrhage, obstruction and perforation. We report a case of 42 year-old Ethiopian male patient presented to the emergency department with sign and symptoms of intestinal obstruction. The patient underwent laparotomy, ileo-ileal intussusception reduced and small bowel mass resection done. The histopathologic examination confirmed malignant small bowel melanoma. The purpose of this case report is to discuss clinical presentation and pathologic features of this rare condition with a review of the current literature.

Keywords: small bowel melanoma, ileo-ileal intussusception
\end{abstract}

\section{Introduction}

Melanomas may arise from the skin or mucosal covering of the respiratory, alimentary, and genitourinary tracts. Mucosal melanomas are rare and accounted for approximately 1 percent of all melanomas [1]. Gastrointestinal tract malignant melanomas are rare form of neoplasm that may be either primary or metastatic. Small bowel metastatic deposits attributed to malignant melanoma originating from skin primary are found in $2-5 \%$ of patients [2]. The melanoma of the small bowel is often asymptomatic. If symptoms are present they are usually nonspecific and can vary from abdominal pain (17-64\%), occult or gross bleeding (24 - 84\%) and weight loss (10 - 47\%) [2]. Diagnosis is often delayed until the illness complicated and become an emergency, such as intestinal hemorrhage, obstruction and perforation [3].

Received: August 2016; Accepted after review: August 2016; Published: September 2016.

*Corresponding author: Mahteme Bekele Muleta, Assistant professor, Department of Surgery, St. Paul's Hospital Millennium Medical College, 1271 Swaziland st, Addis Ababa, Ethiopia.

E-mail: mahtemebekele@gmail.com
In this paper we present a case of small bowel obstruction secondary to ileo-ileal intussusception resulted from metastatic small bowel melanoma. The clinical presentation and pathology description are discussed with review of literatures.

\section{Case report}

A 42 year old Ethiopian male patient presented complaining of crampy abdominal pain of three days duration, followed by frequent fecaloid vomiting and failure to pass feces and flatus of 24 hours duration. On examination, he was acutely sick looking with pulse rate of $96 /$ minute and blood pressure of $110 / 70 \mathrm{mmhg}$. The abdomen was grossly distended with diffuse tenderness, hyper tympanic and hyperactive bowel sound. Laboratory studies showed normal values except a raised white cell count of $13,000 / \mu \mathrm{L}$. Plain abdominal $\mathrm{x}$-ray showed distended bowel loops with multiple air fluid level. With the diagnosis of small bowel obstruction, the patient has briefly resuscitated with intravenous fluids and $1 \mathrm{~g}$ prophylactic ceftriaxone has been administrated. After getting an informed consent, patient underwent laparotomy. The intraoperative 
exploration confirmed the diagnostic with multiple distended bowel loops due to ileo-ileal intussusception $30 \mathrm{~cm}$ from ileocecal valve (figure $1 \mathrm{~A}$ ). The intussusception was reduced and the small bowel segment with charcoal black nodular lesion of $4 \times 3 \mathrm{~cm}$ resected. (Figure 1B).

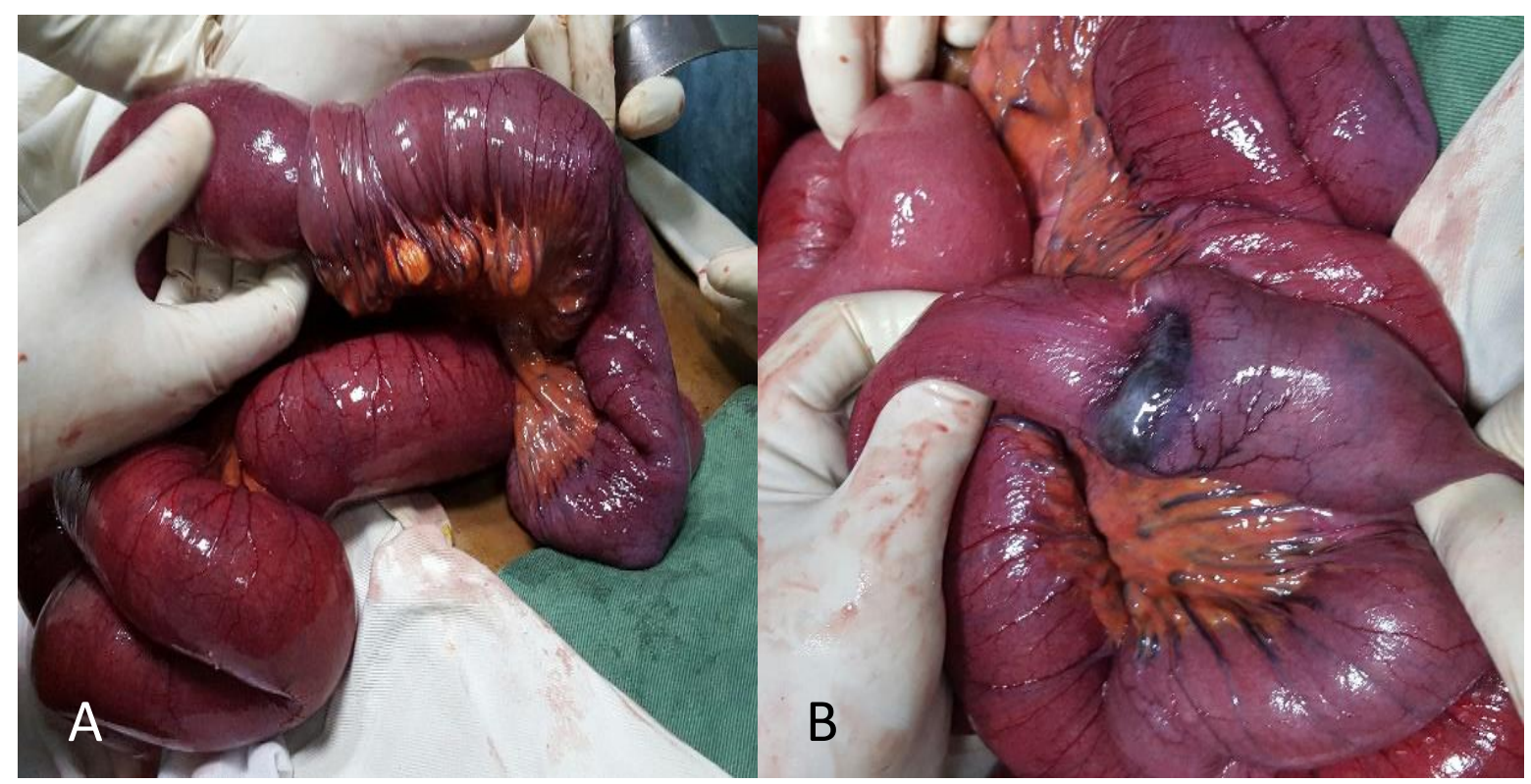

Fig. 1. Intraoperative aspect: A. ileo-ileal intussusception before reduction, B. charcoal black nodular lesion of the ileum.

The histopathology examination confirmed the diagnosis of small bowel melanoma with gross description of small bowel loop $12 \mathrm{~cm}$ long with a blackish nodularity protruding from the subserosal surface (Figure 2A). The cut section showed an intramural, charcoal black, well circumscribed nodularity, measuring $4 \mathrm{x}$ $3.5 \mathrm{~cm}$, covered by a smooth and intact mucosal surface (Figure 2B). Microscopic examination showed submucosal diffuse proliferation of malignant melanocytes (Figure $2 \mathrm{C}$ ). Subsequent postoperative clinical examination detected an acral lentiginous melanoma on the sole of the left foot (Figure 3).

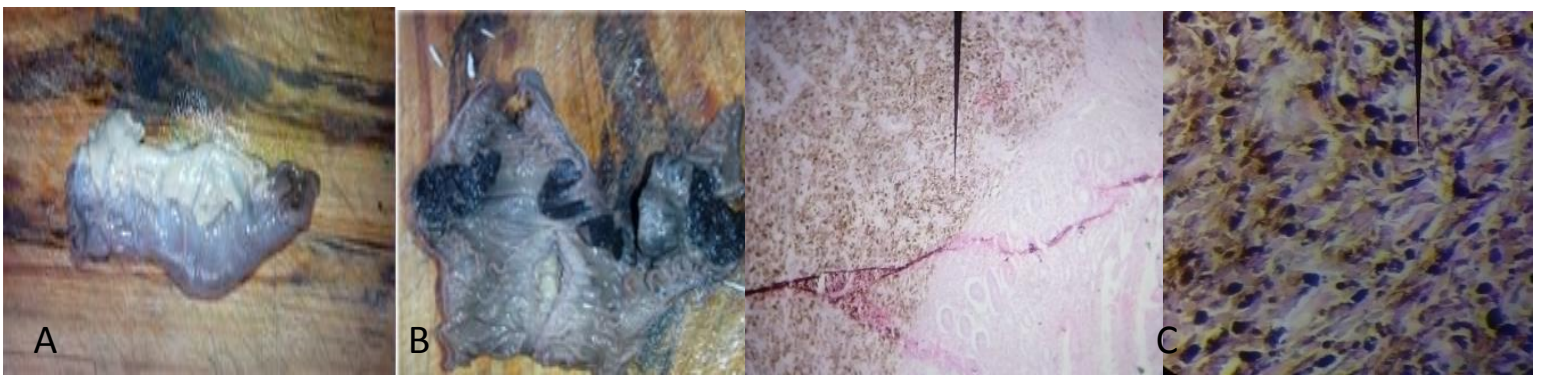

Fig. 2. Histopathologic examination: A. gross appearance of resected small bowel segment, B. cut section showing intermural, charcoal black nodular lesion with intact mucosal surface, C. submucosal diffuse proliferation of malignant melanocytes (HE, x40-100). 


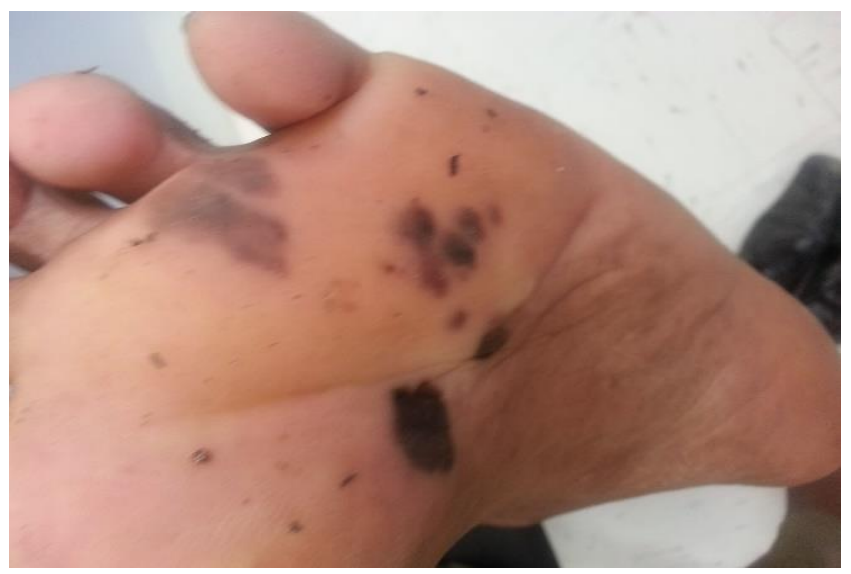

Fig. 3. Acral lentiginous melanoma on the sole of the left foot

\section{Discussions}

Malignant melanoma was reported to be $1 \%$ to $3 \%$ of all gastrointestinal malignancies [4]. Gastrointestinal tract melanomas are mostly metastatic, their most common origin being cutaneous melanoma [5]. A great debate regarding the origin of these tumors has developed, as several cases of primary melanomas of the ileum and the anus have been described $[5,6]$. Some suggest that these neoplasms arise either from the Schwann cells of the gastrointestinal tract or from the neural crest melanoblastic cells, which migrate to the distal ileum through the omphalomesenteric canal [6]. Some researchers consider all gastrointestinal tract melanomas to be metastatic from cutaneous origin, as it is well known fact that some cutaneous melanomas suffer spontaneous regression [7].

Up to $60 \%$ of patients that die from melanoma are found to have metastases at autopsy [8], however, in patients who are alive the metastases detected in less than $4 \%$ of cases. The failure to detect metastasis is due to lack of specific symptoms early in the disease or they are asymptomatic [9]. Sometimes, small bowel melanoma presents with emergency clinical picture due to intestinal obstruction or intestinal intussusception and rarely as bowel perforation [2]. In a review of 13 pathologic specimen of intussusception, lipoma was the most common benign tumor and metastatic malignant melanoma was the most common malignant tumor [10].
Bender et al. defined four different types of metastatic melanoma of the small bowel: cavitary, infiltrating, exoenteric and polypoid ("target" or "bull's eye" lesion). The polypoid pattern is the one most commonly found in small bowel metastasis $[2,10,11]$ which is also true in our case and that may be the reason why the patient developed intussusception; the polypoid lesion acting as lead point.

Different imaging techniques may give a suspicion of intestinal neoplasm; however the definitive diagnosis can be obtained only after surgical exploration. A wide margin intestinal resection including mesentery resection with its lymph nodes remain the treatment of choice. Surgical resection allows getting the final pathologic diagnosis. It is also reported that surgical intervention increased survival significantly; especially when resection is complete on microscopic examination [2, 12].

\section{Conclusions}

A thorough examination of the patient from head to toe is important to detect cutaneous melanoma and rule out metastatic melanoma. Small bowel metastatic melanoma remains a rare cause of small bowel intussusception. Wide margin surgical excision is the main stay of treatment of primary melanoma. Long term follow up and awareness of the natural progression of cutaneous melanoma may help to early detection and modern management of metastatic melanoma. 


\section{References}

1. Chang AE, Karnell LH, Menck HR. The national cancer data base report on cutaneous and non-cutaneous melanoma: a summary of 84,836 cases from the past decade. The American College Of Surgeons Commission On Cancer And The American Cancer Society. Cancer 1998; 83(8):1664-1678.

2. Patti R, Cacciatori M, Guercio G, Territo V, Di Vita G. Intestinal melanoma: A broad spectrum of clinical presentation. IJS Case Reports 2012; 3(8):395-398.

3. Radha S, Afroz T, Ratnakar C, Reddy AK, Satyanarayana G. Small bowel metastasis of malignant melanoma. Indian J Surg 2006; 68:222223.

4. Blecker D, Abraham S, Furth EE, Kochman ML. Melanoma in the gastrointestinal tract. Am J Gastroenterol 1999; 94(12):3427-3433.

5. Schuchter LM, Green R, Fraker D. Primary and metastatic diseases in malignant melanoma of the gastrointestinal tract. Curr Opin Oncol 2000; 12(2):181-185
6. Lens $\mathrm{M}$, Bataille $\mathrm{V}$, Krivokapic $\mathrm{Z}$. Melanoma of the small intestine. Lancet Oncol 2009; 10(5):516-521.

7. Gabali AM, Priebe P, Ganesan S. Primary melanoma of small intestine masquerading as gastrointestinal stromal tumor: A case report and literature review. Am Surg 2008; 74(4):318-321.

8. Manouras A, Genetzakis M, Lagoudianakis $\mathrm{E}$, et al. Malignant gastrointestinal melanomas of unknown origin: Should it be considered primary? World J Gastroenterol 2007; 13(29):4027-4029.

9. Yang WJ, Yoon DS, Lee JJ, Park CJ, Kim $\mathrm{DH}$. Clinical review of the intussusception in adults. J Korean Surg Soc 1998; 55(3):388-393.

10. Bender GN, Maglinte DD, Mc Larney JH, Rex D, Kelvin FM. Malignant melanoma: patterns of metastasis to the small bowel, reliability of imaging studies, and clinical relevance. Am J Gastroenterol 2001; 96(8):2392-2400.

11. Spiridakis KG, Polichronaki EE, Sfakianakis EE, et al. Primary small bowel melanoma. A case report and a review of the literature. G Chir 2015; 36(3):128-132. 